\title{
Molecular Subtypes and Prognostic Factors among Premenopausal and Postmenopausal Thai Women with Invasive Breast Cancer: 15 Years Follow-up Data
}

\author{
Sopit Tubtimhin ${ }^{1}$, Supannee Promthet ${ }^{2,3 *}$, Krittika Suwanrungruang ${ }^{3,4}$, \\ Pongsatorn Supaattagorn ${ }^{5}$
}

\begin{abstract}
Background and Purpose: This study focused on molecular subtypes and prognostic factors for survival of preand post-menopausal breast cancer patients. Methods: A retrospective cohort study was performed on 523 patients with invasive carcinoma of the breast treated at Ubon Ratchathani Cancer Hospital,Thailand from 2002 to 2016. Patient characteristics were collected based on a systematic chart audit from medical records. Prognostic factors were performed by observe survival analysis. A Cox regression model was used to calculate hazard ratios of death, taking into account the age and menopause status, molecular subtype, stage of disease, histological grade, lymphatic and vascular invasion, resection margin, hormone receptor expression, and treatment modality. Results: The median time from the diagnosis of invasive breast cancer to the last follow-up or death was $10.2[95 \% \mathrm{CI}=9.28-11.95]$ years in premenopausal women, and 7.4 [95\% CI $=6.48-8.44]$ years in postmenopausal cases. The overall survival estimates at 5 and 10 years for younger woman of $71.2 \%$ and $51.8 \%$ respectively, appeared slightly better than the $68.3 \%$ and $40.9 \%$ for postmenopausal women $[\mathrm{HRadj}=1.27,95 \% \mathrm{CI}=0.99-1.63]$. In the multivariate analysis, 3 prognostic indicators significantly predicted a worse overall survival in premenopausal patients, triple negative subtype $[\mathrm{HRadj}=$ $6.03,95 \% \mathrm{CI}=1.94-18.74]$, HER2-enriched status $[\mathrm{HRadj}=4.11,95 \% \mathrm{CI}=1.59-10.65]$ and stage $\mathrm{III}[\mathrm{HRadj}=2.73$, $95 \% \mathrm{CI}=1.10-6.79]$. Statistically significant increased risk of death in postmenopausal patients was noted for only chemotherapy after mastectomy [HRadj $=8.76,95 \% \mathrm{CI}=2.88-26.61]$, and for a Luminal B status $[\mathrm{HRadj}=3.55,95 \%$ $\mathrm{CI}=1.47-8.53]$. Conclusion: Postmenopausal women with invasive breast cancer experience a significantly shorter survival than do their premenopausal counterparts. The predictors of worse overall survival were molecular subtype, stage of disease and type of treatment administered.
\end{abstract}

Keywords: Prognostic factors- invasive breast cancer- survival- menopausal status

Asian Pac J Cancer Prev, 19 (11), 3167-3174

\section{Introduction}

The most common type of cancer and the most common cause of cancer-related death in women is breast cancer. Invasive breast cancer is the most common type of malignancy in women worldwide (Wu et al., 2016) and also in Thailand, with estimated incidence rate of 28.5 per 100,000 for the country and 17.0 per 100,000 in Ubon Ratchathani province (W. Imsamran et al., 2015). Incidence rates of breast cancer generally increase with advancing age, although in Asia there may be a peak among women aged approximately 50 years (Jung, 2012). Some $7 \%$ of breast cancers (BC) are diagnosed among women aged below 40 years. Clinical outcomes among young women appear to be especially poor, early age-of-onset increasing the risk of contralateral BC, local and distant recurrence, and subsequent mortality (Brenner et al., 2016). Comorbidities are associated with decreased survival in older women with BC (Dialla et al., 2012).

Included in prognostic factors from previous studies were age, positive nodes, large tumor size (Plichta et al., 2016; Fayer et al., 2016), estrogen receptor negativity and tumor stage at presentation (Hartley et al., 2006), molecular subtype expression, especially triple-negative status (Koca et al., 2014 ; Keegan et al., 2013), HER2-enrichment (Kongsiang et al., 1014), and lymphovascular invasion (Mohammed et al., 2013). One of the biggest challenges in today's breast cancer care is to adjust adjuvant treatment, according to both tumor and patient characteristics, for optimal treatment outcomes. Patients survival remains a significant and challenging medical problem (Dialla et al, 2012; Koca et al, 2014). 
Despite improved knowledge of prognostic factors, there are no data about the compared difference expression molecular subtype and prognostic factors of overall survival among premenopausal and postmenopausal women with $\mathrm{BC}$ in a whole population.

The aim of this study was to investigate differences in molecular subtype and other prognostic factors in premenopausal and postmenopausal women with invasive breast cancer. The hope was that the findings might help in making recommendations for future improvements in early diagnosis and appropriate treatment.

\section{Materials and Methods}

\section{Patients and methods}

\section{Patient Sample selection and Follow-up}

The process for selection of eligible case is illustrated in Figure 1. Of the 1,949 initially considered, 1,077 were excluded because they were not treated in our cancer hospital Of the remaining 869,346 were excluded because they had non-invasive carcinoma (183 patients), experienced menopause before 51 years of age (29 patients), were aged more than 70 years (55 patients), had undergone TAH surgery with BSO (13 patients), were stage IV (25 patients), or had incomplete information (41 patients). The resulting sample cohort (523 patients) consisted of 274 premenopausal and 249 postmenopausal cases.

Patient follow-up commenced at the date of primary invasive breast cancer diagnosis and start of treatment at Ubon Ratchathani Cancer Hospital, Thailand between January 1, 2002 and December 31, 2011. It ended at the date of death, or end of the study (December 31, 2016). Retrospective review of medical records identified characteristics of the patients at presentation, including age, menopause status, molecular subtype - 1) luminal A (estrogen-receptor-positive and/or progesterone-receptor-positive [ER+ and/or $\mathrm{PR}+]$ and negative for human epidermal growth factor receptor 2 [HER2-]), 2) luminal $\mathrm{B}$ (ER+ and/or PR+/ HER2+), 3) HER2-enriched (ER- and PR-/HER2+) and 4) triple-negative (ER- and PR-/HER2-), stage at diagnosis, histological type and grade, histological grading, lymphatic and vascular invasion, resection margin, hormone receptor status, treatment modality and time of the year.

\section{Survival analyses}

The outcome of interest was death due to breast cancer, and analyses of long-term breast cancer-specific survival (15 year) by prognostic factors, for comparison with previous studies. Survival was expressed as the number of years from the date of primary diagnosis to the occurrence of an event, and Kaplan-Meier analyses were performed by menopause status and prognostic factors. Significance was assessed using the log-rank test. A p-value $<0.05$ was considered statistically significant.

Multivariable analysis by molecular subtype was performed using Cox proportional hazard modeling adjusting for classical patient and tumor characteristics (stage of disease, histological grade, presence of lymphatic and vascular invasion, negative resection margin, hormone receptor status, and treatment modality). All factors achieving significance in the univariate analysis were integrated as covariates in the multivariate models. Independent prognostic factors were selected in a stepwise (forward conditional) fashion with use of the maximum likelihood ratio, a p-value of 0.05 or more being adopted as the criterion for exclusion from the final model. All analyses were conducted using STATA version 10.0 (StataCorp LP, 2007).

\section{Ethics}

The study was conducted in accordance with the current revision of the Declaration of Helsinki of the World Medical Assembly, and in conformity with regulations concerning clinical trials under the ICH Good Clinical Practice Guideline. The study protocol and all relevant amendments were reviewed and approved by the Khon Kaen University Ethics Committee for human research (No. HE602003) and approved by the Ubon Ratchathani Cancer Hospital Ethics Committee (No.EC001/2017).

\section{Results}

Follow-up data were available for 523 patients (Table 1). Median follow-up was 9.26 years $(95 \%$ confidence interval $(\mathrm{CI})=8.15-10.4)$; median age was 49 years (range 27-70) with $52.4 \%$ premenopausal $(<51$ years and experiencing menstrual periods) and $47.6 \%$ postmenopausal ( $\geq 5$ 1years and not having experienced any menstrual flow for a minimum of 12 months). The majority of overall patients were molecular subtype luminal A $(31.6 \%, \mathrm{n}=165)$, followed by luminal $\mathrm{B}(15.6 \%, \mathrm{n}=82)$, triple negative $(11.3 \%$, $\mathrm{n}=59)$, and HER2-enriched $(9.9 \%, \mathrm{n}=52)$. Regarding hormone receptors, the majority of $64.6 \%$ were ER positive $(\mathrm{n}=338), 52.9 \%$ were PR negative $(\mathrm{n}=275)$, and $46.6 \%$ were Her- 2 negative $(\mathrm{n}=244) .54 .9 \%$ of the tumors were stage II, and $45.7 \%$ of tumors were grade II (moderately differentiated).

Overall, 70.7\% had a free resection margin $(n=370)$, and $62.5 \%$ demonstrated lymphovascular invasion $(\mathrm{n}=327)$. Almost all underwent surgery $(95.2 \% \mathrm{n}$ $=498), 85.1 \%$ having modified radical mastectomies $(\mathrm{n}=445 / 498)$ and $10.1 \%$ other operations. Some $80.9 \%$ ( $n=423 / 523)$ receive adjuvant chemotherapy after surgery, many also receiving postmastectomy radiation (PMRT) $72.3 \%(\mathrm{n}=378)$ and 58.6 adjuvant endocrine therapy for hormone receptor-positive cancer $(n=198)$.

During the follow-up of the entire cohort, there were 248 deaths from all causes, with $48.7 \%$ premenopausal $(\mathrm{n}=124)$ and $51.2 \%$ postmenopausal $(\mathrm{n}=127)$. Overall, the survival rate was high compared with historical data. Kaplan-Meier estimates for premenopausal Thai women were $71.2 \%$ (95\% confidence interval (CI) $=0.65-0.76)$ at 5 years and $51.8 \%(95 \%$ confidence interval $(\mathrm{CI})=0.44-0.59)$ at 10 years (Figure 2$)$, with a median survival time of 10.6 years $(95 \%$ confidence interval $(\mathrm{CI})=9.28-11.24)$. For postmenopausal Thai women, survival was $68.3 \%$ (95\% confidence interval $(\mathrm{CI})=0.62-0.74)$ at 5 years and $40.9 \%$ (95\% confidence 


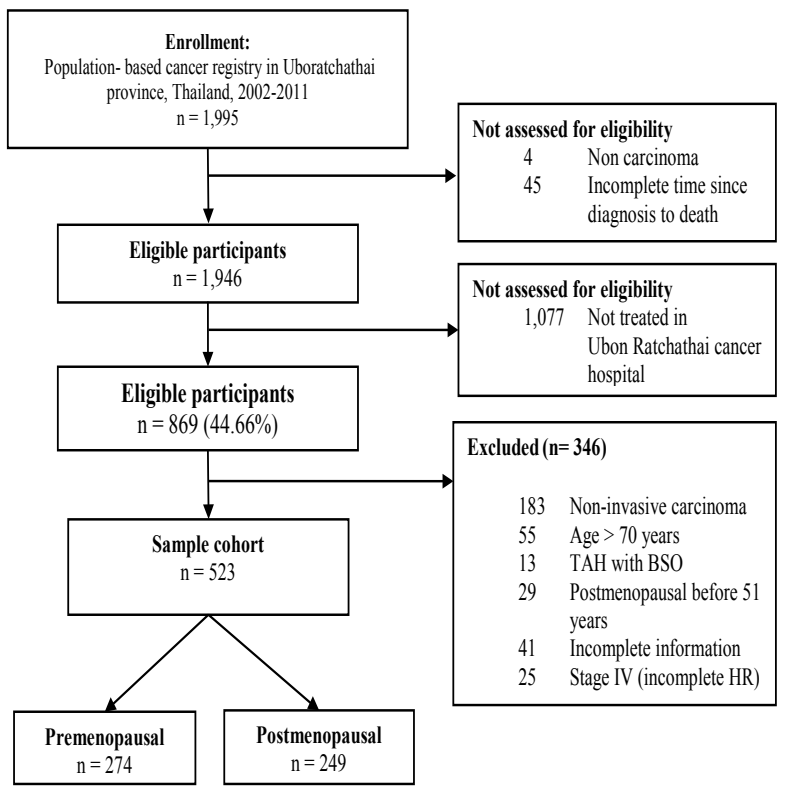

Figure 1. The Sample Selection Process

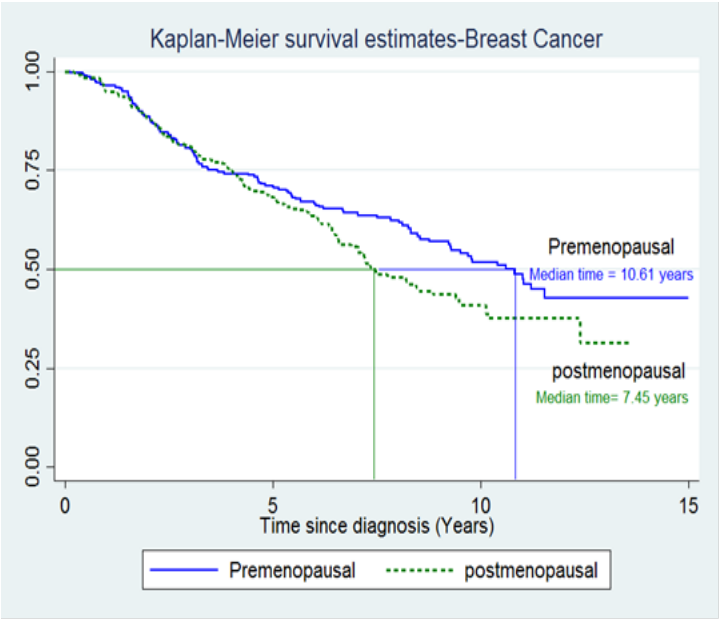

Figure 2. Kaplan-Meier Plot of Overall Breast Cancer Survival by Menopause Status, Thai Women, 20022016, $[$ HRadj $=0.81,95 \%$ confidence interval $(\mathrm{CI})=$ $0.52-1.26$, p-value $<0.036$ ).

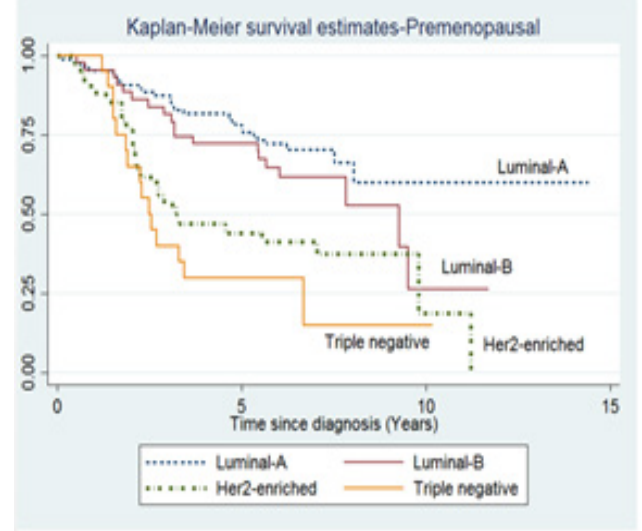

A

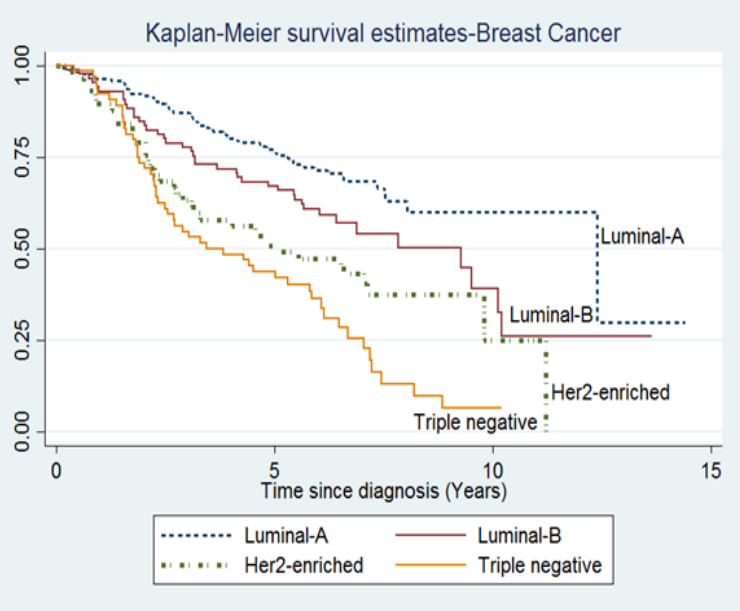

Figure 3. Kaplan-Meier Plot of Overall Breast Cancer Survival by Molecular Subtype, Thai women, 20022016. [The difference of molecular subtype was statistically significant association $(p$-value $=<0.001)$ ]

interval $(\mathrm{CI})=0.33-0.48)$ at 10 years, with a median survival time of 7.45 years $(95 \%$ confidence interval $(\mathrm{CI})$ $=6.47-8.43$ ). Not find statistically significant difference between groups ( $\mathrm{p}$-value $=0.36$ ).

Regarding molecular subtypes, among 523 women with invasive cancer at median follow-up of 7.46 years $(95 \%$ confidence interval $(\mathrm{CI})=6.32-8.59)$, the 5-year survival rates were: Luminal-A $=77.1 \%$, Luminal-B $=67.0 \%$, HER2-enriched $=50.8 \%$ and Triple negative $=43.7 \%$, while 10 -year survival rates were: Luminal-A $=60.0 \%$, Luminal-B $=39.2 \%$, HER2-enriched $=25.0 \%$ and Triple negative $=6.5 \%$. The difference between molecular subtypes groups were statistically significant (Figure 3).

Across the cohort of 274 premenopausal patients with were 124 deaths from all causes, overall survival (OS) at 5 years highest was Luminal-A $=78.2 \%$, Luminal-B $=72.1 \%$, HER 2 -enriched $=44.1 \%$ and Triple negative $=30 \%$, while10 year highest was Luminal-A $=60.1$ $\%$, followed by Luminal-B $=26.4 \%$, HER2-enriched $=18.7 \%$ and Triple negative $=15 \%$. The difference of molecular subtype was statistically significant association $(\mathrm{p}$-value $=<0.001)($ Figure $4 \mathrm{~A})$. For the cohort of 249

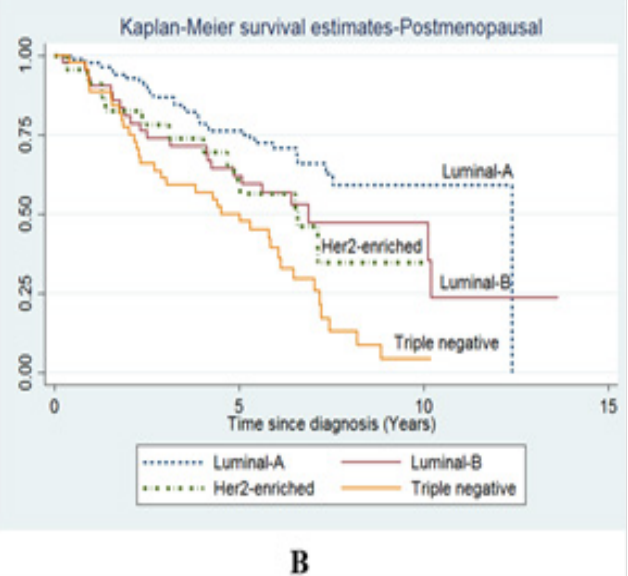

Figure 4. Differential Kaplan-Meier Plot of Breast Cancer Survival by Molecular Subtype among Premenopausal and Postmenopausal, Thai women, 2002-2016. (Premenopausal, p-value $<0.001$ and Postmenopausal, p-value $<0.05$ ) 
Table 1. Demographic and Clinical Characteristics of Breast Cancer Patients $(\mathrm{N}=523)$

\begin{tabular}{|c|c|c|c|c|c|c|}
\hline \multirow[t]{2}{*}{ Characteristics } & \multicolumn{2}{|c|}{ Total } & \multicolumn{2}{|c|}{ Premenopausal } & \multicolumn{2}{|c|}{ Postmenopausal } \\
\hline & Number & $\%$ & Number & $\%$ & Number & $\%$ \\
\hline Overall & 523 & & 274 & 52.39 & 249 & 47.61 \\
\hline \multicolumn{7}{|l|}{ Age } \\
\hline Mean ( \pm Standard Deviation $)$ & $49.6( \pm 9.8)$ & & 41.(9 $9 \pm 5.3)$ & & $58.1 \pm 5.8$ & \\
\hline Median (Min : Max) & $49(27: 70)$ & & $43(27: 50)$ & & $57(51: 70)$ & \\
\hline \multicolumn{7}{|l|}{ Molecular subtypes } \\
\hline Luminal A & 165 & 31.6 & 91 & 33.2 & 74 & 29.7 \\
\hline Luminal B & 82 & 15.6 & 35 & 12.8 & 47 & 18.9 \\
\hline HER2-enriched & 52 & 9.9 & 24 & 8.8 & 28 & 11.3 \\
\hline Triple negative & 59 & 11.3 & 33 & 12 & 26 & 10.4 \\
\hline Unknown & 165 & 31.6 & 91 & 33.2 & 74 & 29.7 \\
\hline \multicolumn{7}{|l|}{ ER } \\
\hline Positive & 338 & 64.6 & 180 & 65.7 & 158 & 63.4 \\
\hline Negative & 185 & 35.4 & 94 & 34.3 & 91 & 36.6 \\
\hline \multicolumn{7}{|l|}{ PR } \\
\hline Positive & 248 & 47.4 & 140 & 51.1 & 108 & 43.4 \\
\hline Negative & 275 & 52.6 & 134 & 48.9 & 141 & 56.6 \\
\hline \multicolumn{7}{|l|}{ Her-2 } \\
\hline Positive & 149 & 28.5 & 83 & 30.3 & 66 & 26.5 \\
\hline Negative & 244 & 46.6 & 114 & 41.6 & 130 & 52.2 \\
\hline Unknown & 130 & 24.9 & 77 & 28.1 & 53 & 21.3 \\
\hline \multicolumn{7}{|l|}{ Stage } \\
\hline Stage I & 33 & 6.3 & 17 & 6.2 & 16 & 6.4 \\
\hline Stage II & 287 & 54.9 & 142 & 51.8 & 145 & 58.2 \\
\hline Stage III & 203 & 38.8 & 115 & 42 & 88 & 35.3 \\
\hline \multicolumn{7}{|l|}{ Histological grade } \\
\hline Grade I (Well differentiated) & 73 & 13.9 & 40 & 14.6 & 33 & 13.2 \\
\hline Grade II (Moderately differentiated) & 239 & 45.7 & 133 & 48.5 & 106 & 43.6 \\
\hline Grade III (Poorly differentiated) & 206 & 39.4 & 100 & 36.5 & 106 & 43.6 \\
\hline Grade IV (Undifferentiated) & 4 & 0.8 & 1 & 0.4 & 3 & 1.2 \\
\hline Unknown & 1 & 0.2 & 0 & 0 & 1 & 0.4 \\
\hline \multicolumn{7}{|l|}{ Resection margin (Free margin) } \\
\hline Yes & 370 & 70.7 & 195 & 71.2 & 175 & 70.3 \\
\hline No & 107 & 20.5 & 59 & 21.5 & 48 & 19.3 \\
\hline Unknown & 46 & 8.8 & 20 & 7.3 & 26 & 10.4 \\
\hline \multicolumn{7}{|l|}{ Lymphovascular invasion } \\
\hline No & 92 & 17.6 & 50 & 18.2 & 42 & 16.9 \\
\hline Yes & 327 & 62.5 & 174 & 63.6 & 153 & 61.4 \\
\hline Unknown & 104 & 19.9 & 50 & 18.2 & 54 & 21.7 \\
\hline \multicolumn{7}{|l|}{ Surgery } \\
\hline Yes & 498 & 95.2 & 264 & 96.4 & 234 & 93.9 \\
\hline No & 25 & 4.8 & 10 & 3.6 & 15 & 6.1 \\
\hline \multicolumn{7}{|l|}{ Postmastectomy radiation } \\
\hline Yes & 378 & 72.3 & 198 & 72.3 & 180 & 72.3 \\
\hline No & 118 & 22.6 & 64 & 23.4 & 54 & 21.7 \\
\hline Unknown & 27 & 5.1 & 12 & 4.3 & 15 & 6 \\
\hline \multicolumn{7}{|l|}{ Adjuvant chemotherapy } \\
\hline Adjuvant & 423 & 80.9 & 225 & 82.1 & 198 & 79.5 \\
\hline Neo-adjuvant & 31 & 3.9 & 11 & 4 & 20 & 8 \\
\hline Unknown & 69 & 13.2 & 38 & 13.9 & 31 & 12.5 \\
\hline
\end{tabular}


DOI:10.31557/APJCP.2018.19.11.3167

Molecular Subtype and Survival of Breast Cancer

Table 1. Continued

\begin{tabular}{|c|c|c|c|c|c|c|}
\hline \multirow[t]{2}{*}{ Characteristics } & \multicolumn{2}{|c|}{ Total } & \multicolumn{2}{|c|}{ Premenopausal } & \multicolumn{2}{|c|}{ Postmenopausal } \\
\hline & Number & $\%$ & Number & $\%$ & Number & $\%$ \\
\hline \multicolumn{7}{|l|}{ Hormone therapy if ER+ $(n=338)$} \\
\hline Yes & 198 & 58.6 & 102 & 56.7 & 96 & 60.8 \\
\hline No & 140 & 41.4 & 78 & 43.3 & 62 & 39.2 \\
\hline \multicolumn{7}{|l|}{ Treatment modality after surgery } \\
\hline Mastectomy + Chemo $+\mathrm{HT}+\mathrm{RT}$ & 158 & 30.2 & 80 & 29.2 & 78 & 31.3 \\
\hline Mastectomy + Chemo + RT & 106 & 20.3 & 61 & 22.3 & 45 & 18 \\
\hline Mastectomy + Chemo + HT & 40 & 7.6 & 16 & 5.8 & 24 & 9.6 \\
\hline Mastectomy+ Chemo & 36 & 6.9 & 21 & 7.7 & 15 & 6.2 \\
\hline Other & 183 & 35 & 96 & 35 & 87 & 34.9 \\
\hline
\end{tabular}

postmenopausal patients 127 deaths from all causes occurred, with overall survival (OS) at 5 years for Luminal- $\mathrm{A}=76.2 \%$, Luminal-B $=61.9 \%$, HER2-enriched $=60.1 \%$ and Triple negative $=50 \%$, and 10 year rates of
Luminal- $\mathrm{A}=59.1 \%$, Luminal-B $=47.1 \%$, HER2-enriched $=34.7 \%$ and Triple negative $=4.3 \%$. The difference of molecular subtype was statistically significant association $(p$-value $=<0.05)$ (Figure 4B).

Table 2. Univariate Analysis of Prognostic Factors Associated on Death in Premenopausal and Postmenopausal Women with Invasive Breast Cancer

\begin{tabular}{|c|c|c|c|c|c|}
\hline Prognostic factors & Median time $(95 \% \mathrm{CI})$ & Person time & $\mathrm{IR} / 100$ & $\mathrm{HR}(95 \% \mathrm{CI})$ & p-value \\
\hline \multicolumn{6}{|l|}{ Premenopausal $(\mathrm{n}=274)$} \\
\hline Molecular subtypes & $7.45(6.32-8.59)$ & & & & $<0.001$ \\
\hline Luminal A & $\mathrm{N} / \mathrm{A}$ & 522 & 5.1 & 1 & \\
\hline Luminal B & $9.06(7.14-11.38)$ & 238 & 7.9 & $1.51(0.84-2.72)$ & \\
\hline HER2-enriched & $3.21(0.64-5.82)$ & 161 & 14.2 & $2.76(1.58-4.84)$ & \\
\hline Triple negative & $2.48(1.93-3.04)$ & 68 & 21.7 & $4.16(2.19-7.87)$ & \\
\hline Stage & & & & & $<0.001$ \\
\hline Stage I & $8.84(8.38-9.30)$ & 138 & 1.4 & 1 & \\
\hline Stage II & N/A & 1099 & 4.3 & $3.08(0.75-12.71)$ & \\
\hline Stage III & $5.43(2.92-7.93)$ & 589 & 12 & $8.78(2.14-35.89)$ & \\
\hline Histological grade & & & & & $<0.001$ \\
\hline Grade I (Well differentiated) & N/A & 315 & 1.4 & 1 & \\
\hline Grade II (Moderately differentiated) & N/A & 955 & 4.8 & $1.06(0.58-1.93)$ & \\
\hline Grade III (Poorly differentiated) & $6.12(4.07-8.35)$ & 552 & 10.8 & $2.47(1.37-4.45)$ & \\
\hline Resection margin (Free margin) & & & & & $<0.001$ \\
\hline Yes & N/A & 1467 & 4.2 & 1 & \\
\hline No & $3.66(1.94-5.39)$ & 285 & 15 & $3.66(2.47-5.43)$ & \\
\hline Lymphovascular invasion & & & & & 0.1 \\
\hline No & $\mathrm{N} / \mathrm{A}$ & 376 & 4.2 & 1 & \\
\hline Yes & $10.61(8.73-12.49)$ & 1156 & 6.5 & $1.54(0.89-2.64)$ & \\
\hline Treatment modality after surgery & & & & & 0.038 \\
\hline Mastectomy + Chemo $+\mathrm{HT}+\mathrm{RT}$ & $10.40(9.12-11.69)$ & 584 & 6.1 & 1 & \\
\hline Mastectomy + Chemo + RT & $8.32(4.55-13.09)$ & 346 & 8.3 & $1.40(0.86-2.28)$ & \\
\hline Mastectomy + Chemo + HT & N/A & 129 & 5.4 & $0.87(0.38-1.96)$ & \\
\hline Mastectomy+ Chemo & $8.12(7.63-8.60)$ & 150 & 8.3 & $1.45(0.77-2.74)$ & \\
\hline \multicolumn{6}{|l|}{ Postmenopausal $(\mathrm{n}=249)$} \\
\hline Molecular subtypes & & & & & $<0.001$ \\
\hline Luminal A & $12.38(12.14-12.63)$ & 497 & 5.8 & 1 & \\
\hline Luminal B & $6.89(3.76-10.01)$ & 225 & 9.6 & $1.64(0.94-2.87)$ & \\
\hline HER2-enriched & $6.57(5.91-7.23)$ & 122 & 10.6 & $1.90(0.98-3.67)$ & \\
\hline Triple negative & $5.02(3.01-7.03$ & 192 & 18.1 & $3.30(2.01-5.42)$ & \\
\hline
\end{tabular}


Table 2. Continued

\begin{tabular}{|c|c|c|c|c|c|}
\hline Prognostic factors & Median time $(95 \% \mathrm{CI})$ & Person time & $\mathrm{IR} / 100$ & $\operatorname{HR}(95 \% \mathrm{CI})$ & p-value \\
\hline Stage & & & & & $<0.001$ \\
\hline Stage I & $8.84(8.38-9.30)$ & 108 & 6.4 & 1 & \\
\hline Stage II & $\mathrm{N} / \mathrm{A}$ & 1006 & 5.5 & $0.88(0.40-1.93)$ & \\
\hline Stage III & $4.90(3.76-6.04)$ & 404 & 15.8 & $2.83(1.29-6.22$ & \\
\hline Histological grade & & & & & 0.001 \\
\hline Grade I (Well differentiated) & $10.21(10.05-10.36)$ & 245 & 4.8 & 1 & \\
\hline Grade II (Moderately differentiated) & $8.44(6.22-10.88)$ & 698 & 6.7 & $1.40(0.74-2.65)$ & \\
\hline Grade III (Poorly differentiated) & $6.41(5.58-7.24)$ & 557 & 11.4 & $2.46(1.32-4.58)$ & \\
\hline Resection margin (Free margin) & & & & & $<0.001$ \\
\hline Yes & $10.13(8.20-13.62)$ & 1198 & 5.8 & 1 & \\
\hline No & $5.81(4.70-6.92)$ & 237 & 14.7 & $2.84(1.87-4.30)$ & \\
\hline Lymphovascular invasion & & & & & 0.146 \\
\hline No & N/A & 218 & 5.3 & 1 & \\
\hline Yes & $7.45(6.16-8.75)$ & 962 & 7.9 & $1.48(0.85-2.57)$ & \\
\hline Treatment modality after surgery & & & & & $<0.001$ \\
\hline Mastectomy + Chemo $+\mathrm{HT}+\mathrm{RT}$ & $9.47(7.2-11.68)$ & 494 & 5.6 & 1 & \\
\hline Mastectomy + Chemo + RT & N/A & 333 & 6.8 & $1.28(0.73-2.23)$ & \\
\hline Mastectomy + Chemo + HT & $7.33(6.67-7.99)$ & 151 & 7.2 & $1.28(0.63-2.58)$ & \\
\hline Mastectomy+ Chemo & $2.51(0.31-4.72)$ & 42 & 27.9 & $5.29(2.68-10.45)$ & \\
\hline
\end{tabular}

Table 3. Multivariate Cox Regression Analysis of Prognostic Factors Associated on Death in Premenopausal and Postmenopausal Women with Invasive Breast Cancer

\begin{tabular}{|c|c|c|c|c|c|c|}
\hline \multirow[t]{2}{*}{ Prognostic factors } & \multicolumn{3}{|c|}{ Premenopausal } & \multicolumn{3}{|c|}{ Postmenopausal } \\
\hline & $\begin{array}{c}\text { Crude HR } \\
(95 \% \mathrm{CI})\end{array}$ & $\begin{array}{c}\text { Adjusted HR } \\
(95 \% \mathrm{CI})\end{array}$ & $\begin{array}{c}\mathrm{p}- \\
\text { value }\end{array}$ & $\begin{array}{c}\text { Crude HR } \\
(95 \% \mathrm{CI})\end{array}$ & $\begin{array}{c}\text { Adjusted HR } \\
(95 \% \mathrm{CI})\end{array}$ & $\begin{array}{c}\mathrm{p}- \\
\text { value }\end{array}$ \\
\hline Molecular subtypes & & & $<0.001$ & & & $<0.05$ \\
\hline Luminal A & 1 & 1 & & 1 & 1 & \\
\hline Luminal B & $1.51(0.84-2.72)$ & $1.87(0.87-4.06)$ & & $1.64(0.94-2.87)$ & $3.55(1.47-8.53)$ & \\
\hline HER2-enriched & $2.76(1.58-4.84)$ & $4.11(1.59-10.65)$ & & $1.90(0.98-3.67)$ & $1.36(0.43-4.27)$ & \\
\hline Triple negative & $4.16(2.19-7.87)$ & $6.03(1.94-18.74)$ & & $3.30(2.01-5.42)$ & $2.11(0.82-5.44)$ & \\
\hline Stage & & & 0.034 & & & 0.153 \\
\hline Stage I & 1 & 1 & & 1 & 1 & \\
\hline Stage II & $3.08(0.75-12.71)$ & $1.82(0.89-3.08)$ & & $0.88(0.40-1.93)$ & $0.34(0.33-3.62)$ & \\
\hline Stage III & $8.78(2.14-35.89)$ & $2.73(1.10-6.79)$ & & $2.83(1.29-6.22$ & $0.82(0.60-11.40)$ & \\
\hline Histological grade & & & 0.315 & & & 0.526 \\
\hline Grade I & 1 & 1 & & 1 & 1 & \\
\hline Grade II & $1.06(0.58-1.93)$ & $1.17(0.42-3.20)$ & & $1.40(0.74-2.65)$ & $0.57(0.19-1.73)$ & \\
\hline Grade III & $2.47(1.37-4.45)$ & $1.67(0.53-5.27)$ & & $2.46(1.32-4.58)$ & $1.04(0.35-3.08)$ & \\
\hline Resection margin (Free margin) & & & 0.31 & & & 0.821 \\
\hline Yes & 1 & 1 & & 1 & 1 & \\
\hline No & $3.66(2.47-5.43)$ & $1.69(0.58-4.89)$ & & $2.84(1.87-4.30)$ & $1.26(0.36-4.38)$ & \\
\hline Lymphovascular invasion & & & 0.323 & & & 0.863 \\
\hline No & 1 & 1 & & 1 & 1 & \\
\hline Yes & $1.54(0.89-2.64)$ & $1.47(0.59-3.72)$ & & $1.48(0.85-2.57)$ & $1.05(0.44-2.47)$ & \\
\hline Treatment modality & & & 0.3 & & & 0.001 \\
\hline Mastectomy + Chemo $+\mathrm{HT}+\mathrm{RT}$ & 1 & 1 & & 1 & 1 & \\
\hline Mastectomy + Chemo + RT & $1.40(0.86-2.28)$ & $0.88(0.40-1.93)$ & & $1.28(0.73-2.23)$ & $1.77(0.78-4.01)$ & \\
\hline Mastectomy + Chemo $+\mathrm{HT}$ & $0.87(0.38-1.96)$ & $0.78(0.28-2.20)$ & & $1.28(0.63-2.58)$ & $1.56(0.42-5.77)$ & \\
\hline Mastectomy+ Chemo & $1.45(0.77-2.74)$ & $2.16(0.71-5.71)$ & & $5.29(2.68-10.45)$ & $8.76(2.88-26.61)$ & \\
\hline
\end{tabular}


On univariate analysis, statistically significant risk of death in both premenopausal and postmenopausal invasive breast cancer was associated with molecular subtypes, stage of disease, histological grade, resection margin status, lymphovascular invasion and treatment modality (Table 2). With the multivariate proportional hazard model including these variables, only molecular subtypes and stage of disease remained significant in predicting a worse OS in premenopausal patients (by Cox regression, $\mathrm{p}$-values $<0.001$ and 0.034 respectively ), highest risk compared to Luminal $\mathrm{A}$ was with Triple negative $[\mathrm{HRadj}=$ $6.03,95 \%$ confidence interval $(\mathrm{CI})=1.94-18.7]$, followed by HER2-enriched [HRadj $=4.11,95 \%$ confidence interval $(\mathrm{CI})=1.59-10.65]$ and Stage III as compared to Satge I $[\mathrm{HRadj}=2.73,95 \%$ confidence interval $(\mathrm{CI})=$ 1.10-6.79]. Statistically significant for postmenopausal patients were molecular subtype, Luminal B compared to Luminal A [HRadj $=3.55,95 \%$ confidence interval $(C I)=1.47-8.53$; Table 3] and treatment modality after mastectomy, chemotherapy only compared to chemotherapy +HT+RT $[$ HRadj $=8.76,95 \%$ confidence interval $(\mathrm{CI})=2.88-26.61, \mathrm{p}-$ value $<0.001]$. We found poorer survival among postmenopausal than premenopausal $\mathrm{BC}[\mathrm{HRadj}=1.27,95 \%$ confidence interval $(\mathrm{CI})=0.99-1.63]$ (data not shown).

\section{Discussion}

The results of the present study showed that risk of death overall in younger women (27 to 50 years of age: premenopausal) was marginally greater than in postmenopausal women (51 to 70 years of age) (not statistically significant) after adjustment for all known prognostic factors. Bharat et al., (2009) previously reported that younger women were more likely to die from breast cancer than older women. Age and menopausal status have no significant effect on overall survival of breast cancer patients in Pakistan (Mahmood et al., 2015).The reasons for the improved outcomes observed in our study are likely multifactorial. Such as better staging also allowed us to exclude patients with metastatic disease at diagnosis who may have been included in prior series of young breast cancer patients, the benefit of digital mammography for early screening as well as the increasing use of breast MRI in their preoperative workup (data not shown).

Several studies published before 2010 found young age at diagnosis to be an independent predictor of poor prognosis (Bharat et al., 2009 ; Gnerlich et al., 2009). After that, outcomes for women with breast cancer at age $<40$ years have improved (Plichta et al., 2016). For example, Beadle et al., (2009) found OS to be $64.6 \%$ at 10 years, Anders al., (2009) found overall mortality (RR $=1.50, \mathrm{P}<.04)$. Plichta et al., (2016) found OS to be 86.5 $\%$ at 10 years. In our study, OS was $51.8 \%$ at 10 years. Which, suggesting that recent improvements in diagnosis and treatment have translated into improved outcomes for young women. And suggesting that $\mathrm{BC}$ survival in the developed countries has continually improved and has largely been accredited to the use of mammography, advanced surgical procedures, and adjuvant therapies. These technological and diagnostic advances, suggesting important opportunities for early detection and treatment and be the cause of increased opportunities for improved survival.

Regarding molecular subtypes on overall survival, we found that survival was highest for patients with luminal-A cancers, followed by those with luminal B cancers. This confirms the better outcomes in hormone-receptor-positive cancers reported in other jurisdictions (Bauer et al., 2007; Yang et al., 2007; Kennecke et al., 2010; Voduc et al., 2010; Fallahpour et al., 2017).

Younger age with molecular subtype expression has been shown in several studies to be an independent predictor of adverse outcome. Keegan et al., (2013) found young women have poorer survival after breast cancer than do older women 40 to 64 years, adolescent and young adult 15 to 39 years. Women diagnosed with HR-/HER + and triple-negative breast cancer experienced a 1.6-fold and 2.7-fold increased risk of death, respectively, from all causes [HR-/HER + hazard ratio: 1.55; 95\% confidence interval (CI): 1.10 to 2.18 ; triple-negative HR: 2.75 ; $95 \%$ CI, 2.06 to 3.66] and breast cancer [HR-/HER + hazard ratio: 1.63 ; $95 \% \mathrm{CI}, 1.12$ to 2.36 ; triple-negative hazard ratio: $2.71 ; 95 \% \mathrm{CI}, 1.98$ to 3.71 ]. In our studies, triple negative was highest of related [HRadj 6.03; 95\% CI =1.94-18.74], follow by HER2-enriched [HRadj 4.11; $95 \% \mathrm{CI}=1.59-10.65]$. Suggesting that worse short-term survival for HR-/HER2+ (HER2-enriched) and triplenegative breast cancers is consistent with prior studies that had 5 or 10 years of follow up. Earlier studies in Thailand TNs demonstrated significantly higher tumor grade, mitotic count, Ki-67 index, p53 and vimentin and decreased overall survival compared with nonTN (Chuangsuwanich et al., 2014). HER-2 and basal-like subtypes were likely to have high tumor grade, HER-2 subtype larger, had higher number of nodal involvement (Chuthapisith et al., 2012).

For postmenopausal $\mathrm{BC}$, the major independent prognostic factors associated with increased risk of death were treatment modality after mastectomy received only chemotherapy had a significantly higher risk of death $[$ HRadj $=8.76,95 \% \mathrm{CI}=2.88-26.61]$, follow by Luminal $\mathrm{B}$ [HRadj $=3.55,95 \% \mathrm{CI}=1.47-8.53]$.

Our findings provide evidence to suggest that an interaction between premenopausal status breast cancer and molecular subtype suggests high risk triple-negative breast cancers, which are less common, have substantially worse prognosis than Intermediate risk HER2-enriched (ER-and PR-/HER2+) Intermediate risk Luminal B (ER+ and/or PR+/HER2+) and low risk Luminal A[ER+ and/or $\mathrm{PR}+]$ breast cancer. For postmenopausal status, high risk in Luminal B (ER+ and/or PR+/HER2+).

The strength of this study is that the analyses were done in a heterogeneous and exhaustive group of patients, using data from systematic chart audit of demographic features and medical record from Ubon Ratchathani Cancer Hospital. Therefore, the results could be considered representative of $\mathrm{BC}$ patients living in the department during this period.

Limitation of the study for 523 patients included in this study were those for whom a complete set of data was available. The exclusion of those with incomplete data is 
potential source of bias. However, the characteristics of those excluded due to incomplete data were similar to and not statistically different from those with complete data.

In conclusions, postmenopausal women of invasive breast cancer experience a significantly shorter survival than those with premenopausal women. Despite the fact that prognostic factors in premenopausal BC was increased risk of death higher than postmenopausal BC. Moreover the results of this study showed that there are numerous predictors of a worse OS, and that the most important were molecular subtype, stage of disease and type of treatment administered.

\section{Conflict of interest}

The authors disclosed no potential conflicts of interest related to the current study.

\section{Acknowledgments}

The authors would like to thank all the staff at Ubon Ratchathani Cancer Hospital, Department of Medical Services, Thailand for their kind support during the data collection process.

\section{References}

Anders CK, Johnson R, Litton J, Phillips M, Bleyer A (2009). Breast cancer before age 40 years. Semin Oncol, 36, 237-49.

Bauer KR, Brown M, Cress RD, et al (2007). Descriptive analysis of estrogen receptor(ER)negative, progesterone receptor (PR)-negative, and HER2-negative invasive breast cancer, the so-called triple-negative phenotype: a population-based study from the California Cancer Registry. Cancer, 109, 1721-8.

Beadle BM, Woodward WA, Tucker SL, et al (2009). Ten-year recurrence rates in young women with breast cancer by locoregional treatment approach. Int J Radiat Oncol Biol Phys, 73, 734-44.

Bharat A, Aft RL, Gao F, Margenthaler JA (2009). Patient and tumor characteristics associated with increased mortality in young women ( $<$ or $=40$ years) with breast cancer. $J$ Surg Oncol, 100, 248-51.

Brenner DR, Brockton NT, Kotsopoulos J, et al (2016). Breast cancer survival among young women: a review of the role of modifiable lifestyle factors. Cancer Causes Control, 27, 459-72.

Chuangsuwanich T, Pongpruttipan T, P OC, et al (2014). Clinicopathologic features of breast carcinomas classified by biomarkers and correlation with microvessel density and VEGF expression: a study from Thailand. Asian Pac J Cancer Prev, 15, 1187-92.

Chuthapisith S, Permsapaya W, Warnnissorn M, et al (2012). Breast cancer subtypes identified by the ER, PR and HER-2 status in Thai women. Asian Pac J Cancer Prev, 13, 459-62.

Dialla PO, Dabakuyo TS, Marilier S, et al (2012). Population-based study of breast cancer in older women: prognostic factors of relative survival and predictors of treatment. BMC Cancer, 12, 472 .

Fallahpour S, Navaneelan T, De P, Borgo A (2017). Breast cancer survival by molecular subtype: a population-based analysis of cancer registry data. CMAJ Open, 5, 734-9.

Fayer VA, Guerra MR, Cintra JR, Bustamante-Teixeira MT (2016). Ten-year survival and prognostic factors for breast cancer in the southeast region of Brazil. Rev Bras Epidemiol OUT-DEZ, 19, 766-78.
Gnerlich JL, Deshpande AD, Jeffe DB, et al (2009). Elevated breast cancer mortality in women younger than age 40 years compared with older women is attributed to poorer survival in early-stage disease. J Am Coll Surg, 208, 341-7.

Hartley MC, McKinley BP, Rogers EA, et al (2006). Differential expression of prognostic factors and effect on survival in young $(<$ or $=40)$ breast cancer patients: a case-control study. Am Surg, 72, 1189-94.

Jung SY, Rosenzweig M, Linkov F, et al (2012). Comorbidity as a mediator of survival disparity between younger and older women diagnosed with metastatic breast cancer. Hypertension, 59, 205-11.

Keegan TH, Press DJ, Tao L, et al (2013). Impact of breast cancer subtypes on 3-year survival among adolescent and young adult women. Breast Cancer Res, 15, R95.

Kennecke H, Yerushalmi R, Woods R, et al (2010). Metastatic behavior of breast cancer subtypes. J Clin Oncol, 28, 3271-7.

Koca B, Kuru B, Karabicak I, Yuruker SS, Ozen N (2014). Prognostic factors affecting disease-free survival in patients at age 35 or younger with invasive breast cancer. Ann Ital Chir, 85, 249-53.

Kongsiang A, Tangvoraphonkchai V, Jirapornkul C, et al (2015). Survival time and molecular subtypes of breast cancer after radiotherapy in Thailand. Asian Pac J Cancer Prev, 15, 10505-8.

Mahmood H, Faheem M, Mahmood S, Sadiq M, Irfan J (2015). Impact of age, tumor size, lymph node metastasis, stage, receptor status and menopausal status on overall survival of breast cancer patients in Pakistan. Asian Pac J Cancer Prev, 16, 1019-24.

Mohammed ZM, McMillan DC, Edwards J, et al (2013). The relationship between lymphovascular invasion and angiogenesis, hormone receptors, cell proliferation and survival in patients with primary operable invasive ductal breast cancer. BMC Clin Pathol, 13, 31.

Plichta JK, Rai U, Tang R, et al (2016). Factors associated with recurrence rates and long-term survival in women diagnosed with breast cancer ages 40 and younger. Ann Surg Oncol, 23, 3212-20.

Voduc KD, Cheang MC, Tyldesley S, et al (2010). Breast cancer subtypes and the risk of local and regional relapse. J Clin Oncol, 28, 1684-91.

W. Imsamran AC, Wiangnon S, Pongnikorn D, Suwanrungrung K , Sangrajrang S (2015). Cancer in Thailand Vol. VIII, 2010-2012. Bangkok: New Thammada Press (Thailand) Co., Ltd. 2015.

Wu X, Sun L, Wang X, et al (2016). Breast cancer invasion and metastasis by mPRalpha through the PI3K/Akt signaling pathway. Pathol Oncol Res, 22, 471-6.

Yang XR, Sherman ME, Rimm DL, et al (2007). Differences in risk factors for breast cancer molecular subtypes in a population-based study. Cancer Epidemiol Biomarkers Prev, 16, 439-43.

This work is licensed under a Creative Commons AttributionNon Commercial 4.0 International License. 